\title{
Clinical Characterization of COVID-19
}

\author{
Hassan Naji
}

\begin{abstract}
The world is on the brink of a pandemic due to the Novel coronavirus-19. The World Health Organization (WHO) urged countries all over the world to take necessary measures to control then new coronavirus disease (COVID-19) and further limit the overwhelmingly number of people infected and falling ill to the disease. It is widely known that COVID-19 affects the lung causing pneumonia with characteristic ground-glass opacity. Few research studies outline the effect of the Novel coronavirus on other parts of the body such as stomach, liver, and kidneys. Interestingly, COVID-19 causes a cytokine storm that overwhelms the body leading to inhibition of body's cellular immune responses by the virus through lowering the white blood cells count specifically lymphocytes, and platelets count. This paper summarizes the effect of COVID-19 on various body organs.
\end{abstract}

Index Terms-2019-nCoV, SARS-CoV-2, Coronavirus, Novel coronavirus, COVID-19, MERS-CoV, SARS-CoV, Lung Diseases, Pneumonia, Gastric Ulcer, Lung Cells, Acute Respiratory Disease, ARD, Cytokines Storm, Subpleural Ground-Glass Opacity, Multi-Lobular Ground-Glass Opacity, Lymphopenia, Receptor Binding Domain, RBD, Vomiting, Diarrhea, Pregnancy and COVID-19, Liver Damage, AST, ALT, Renal Damage.

\section{INTRODUCTION}

On January 7, 2020, Chinese health authorities confirmed a cluster of cases of pneumonia to be associated with a virus, the 2019-nCoV. The virus was renamed SARS-CoV-2 (CDC, 2020). WHO declared the virus a public health emergency of international concern, and health departments around the world started scrambling to control the virus (Guan et al., 2020). The epidemiological association of patients with viral pneumonia in December 2019, was found to be with Huanan seafood market in Wuhan, China. In addition, the virus has been identified in several other countries. Infection has been reported in family clusters and medical workers. Also, human to human transmission has been reported (Lu et al., 2020). As of January 31, 2020, a total of 9692 cases of $2019-\mathrm{nCoV}$ infection in China have been confirmed. As of February 5, 2020, 24,554 laboratoryconfirmed cases have been documented worldwide. On March 11, 2020, the WHO declared COVID-19 as a pandemic. According to the WHO briefing on March 13, 2020, more than 120,000 cases of COVID-19 with over 45,00 deaths in 100 countries have been reported (WHO, 2020).

The phylogenetic analysis of the virus demonstrated that 2019-nCoV is a beta-coronaviruses like severe acute respiratory syndrome-coronavirus (SARS-CoV) and Middle

Published on March 24, 2020.

Hassan Naji, Purdue University Northwest, USA.

(e-mail: hnaji@pnw.edu )
East respiratory syndrome-coronavirus (MERS-CoV). The complete genomic sequence of $2019-\mathrm{nCoV}$ is most closely related to bat-SL-CoVZC45 and another beta-coronavirus of bat origin, bat-SL-CoVZXC21 (Chen et al., 2020). On February 11, the International Committee on Taxonomy of Viruses (ICTV) announced the new official name of the virus that causes COVID-19 as Severe Acute Respiratory Syndrome coronavirus 2 or SARS-CoV-2 (Gorbalenya et al., 2020).

The clinical picture of SARS-CoV-2 is largely similar to SARS-CoV. The early clinical manifestations include fever, non-productive cough, myalgias, diarrhea, dyspnea, nausea and vomiting. Laboratory findings provide evidence of infection including lymphopenia, thrombocytopenia, elevated C-reactive protein, liver enzymes, blood urea nitrogen, creatinine and lactate dehydrogenase (LDH) levels. On chest CT patients show ground-glass opacity and multiple infiltrates. Patients having an underlying disorder present with severe clinical outcomes and require careful monitoring and treatment. Various complications are reported including acute respiratory distress syndrome (ARDS), acute respiratory disease (ARD), respiratory failure, kidney failure, and cytokine storm leading to death (Huang et al., 2020).

\section{EFFECT ON THE LUNGS}

SARS-CoV-2 primarily enters and infects human lung cells. The clinical effects of SARS-CoV-2 are similar to those of SARS-CoV, and MERS-CoV, which cause severe respiratory syndrome in humans. Initially, the outbreak of SARS-CoV-2 in Wuhan, Hubei, China, started as a series of pneumonia cases without a known case and clinical picture majorly resembled community acquired viral pneumonia. The respiratory specimens, including, bronchoalveolar lavage fluid, bronchial aspirates, nasal and pharyngeal swabs, or sputum were taken from the patients and tested using real-time RT-PCR assays, for common viruses like avian influenza, respiratory syncytial viruses, adenovirus, influenza, parainfluenza virus, MERS-CoV, and SARS$\mathrm{CoV}$, which were all negative. The specimens from pharyngeal and nasal swabs of the patients were positive for 2019-nCoV real-time RT-PCR and high-throughput sequencing (Huang et al., 2020). The prominent symptoms of infection are majorly fever, shortness of breath, dry mucous membranes and persistent dry cough. Other symptoms include myalgias, headache, chest pain, and diarrhea. Radiologic evidence of pneumonia and nonproductive cough are among the initial symptoms of infection. In severe cases, acute cardiac injury, acute respiratory distress syndrome (ARDS), respiratory failure and death can occur, and patients require invasive ventilation, oxygen therapy or extracorporeal membrane 
oxygenation (Wang et al., 2020).

At presentation, the hallmark of SARS-CoV-2 infection on radiologic investigation include bilateral patchy shadowing and ground glass opacity on chest computed tomography (Figure 1). Infected patients having an underlying disease like, hypertension, diabetes, cardiovascular disease, malignancy, or chronic obstructive pulmonary disease are categorized into severe cases. In nonsevere cases, transverse chest $\mathrm{CT}$ imaging shows subpleural and multi-lobular ground-glass opacity and consolidation, however, in severe cases, there is evidence of multi-lobular sub-segmented consolidation (Guan et al., 2020).

A: CT images on day-5 after onet of symptoms
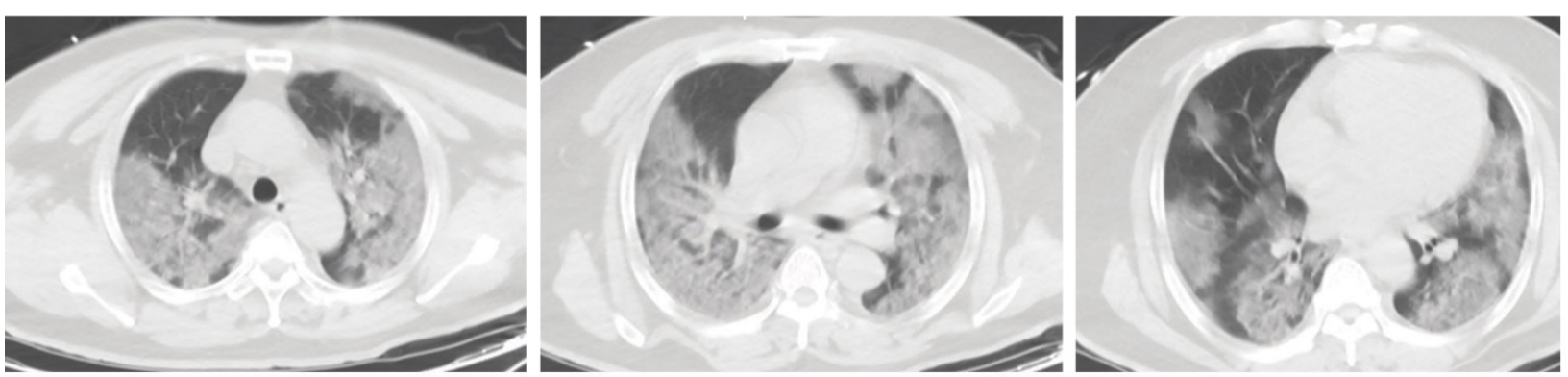

B: CT images on day-19 after treatment after onset of symptoms
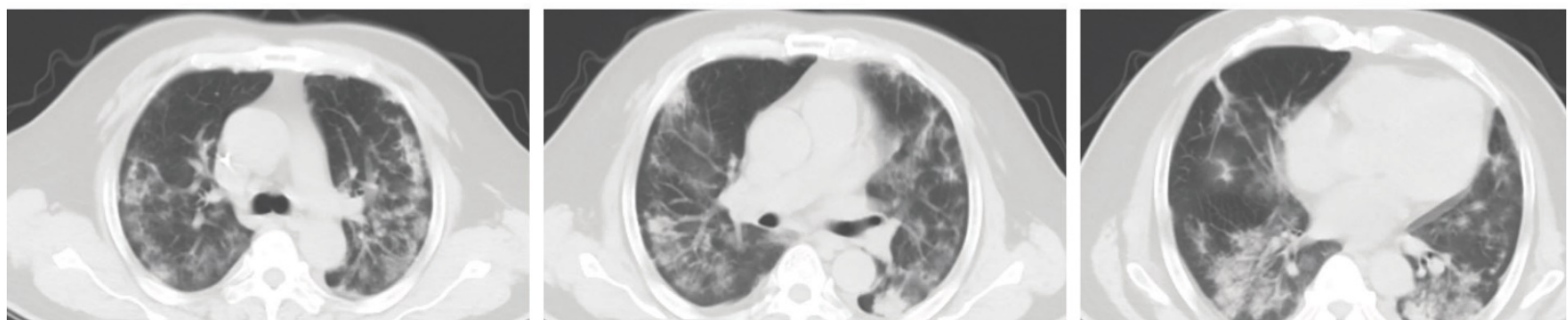

Fig. 1: Chest CT images of a 52-year old patient infected with 2019-nCoV. A: shows ground glass opacity in both lungs B: shows absorption of bilateral ground glass. CT: computed tomography 2019-nCoV: 2019-novel coronavirus. Adopted from Guan et al., 2020

In a study by Nanshan Chen et al., 75\% patients with SARS-CoV-2 infection showed bilateral pneumonia while $25 \%$ showed unilateral pneumonia on chest X-ray and CT. Also, 14\% patients showed ground glass opacity and multiple mottling and $1 \%$ showed pneumothorax. Patients can also present with organ dysfunction including acute respiratory syndrome, acute respiratory injury, septic shock and also with ventilator-associated pneumonia (Chen et al., 2020).

SARS-CoV-2, like SARS-CoV, is a beta-coronavirus. The overall sequence of spike protein of SARS-CoV-2 is $76-78 \%$ similar to SARS-CoV spike. This spike protein binds to specific host receptor, following fusion with host membranes and finally gains entry into the host cells and cause infection. The receptor binding domain (RBM) on SARS-CoV spike binds to specific host receptor angiotensin-converting enzyme 2 (ACE2), and enters the cells. Just like SARS-CoV, SARS-CoV-2 also uses this ACE2 receptor and enters and infects primarily host lung cells, which leads to clinical symptoms like dyspnea, productive cough, and abnormal radiologic findings on chest X-ray and CT. If unchecked, the COVID-19 worsens and shortness of breath occurs while coughing. In severe cases, patients require ventilatory support when their oxygen saturation is not maintained properly (Wan, Shang, Graham, Baric, \& Li, 2020).

\section{The Gastrointestinal Tract}

Patients infected with SARS-CoV-2 also presented with less common symptoms, including vomiting, diarrhea, anorexia, fatigue, dizziness, abdominal discomfort and nausea, indicating digestive tract abnormalities. In several cases, significant proportion of patients presented with history of nausea, diarrhea and vomiting without prior development of fever, dyspnea, and abnormal radiologic findings (Holshue et al., 2020). In some cases, fever and diarrhea are the only initial presenting symptoms. After the onset of symptoms, the patient may develop severe peptic ulcer in rare cases. In such patients, SARS-CoV-2 can be detected directly at the bleeding site and esophageal erosion. It can also be detected in gastrointestinal secretions and can be transmitted via these secretions. Patients usually develop gastrointestinal symptoms during second week of illness. The consistency of stool is usually watery. Diarrhea causes water and electrolyte loss, which leads to volume reduction and electrolyte disturbances. Soon after the progression of diarrhea, SARS-CoV-2 can be detected in stool samples by real time RT-PCR. The diarrhea is usually self-limiting, and no fatalities have been reported due to diarrhea (Wang et al., 2020).

\section{BLOOD STREAM}

The complete blood count examination of patients infected with COVID-19 revealed lymphopenia (82.1\%), thrombocytopenia (36.2\%), and leucopenia (33.7\%). In addition, increased levels of C-reactive protein, alanine aminotransferase, creatine kinase, creatine, aspartate aminotransferase and D-dimer were also observed (Guan et al., 2020). The reduced number of lymphocytes in patients suggests that SARS-CoV-2 mainly infects lymphocytes, 
specifically T-lymphocytes, like the SARS-CoV. The virus transmits from one person to other through respiratory mucosa, and enters other cells which induce increased cytokine production in the body, activates a series of immune responses and change the counts of white blood cells in the blood. Some studies suggest that reduced number of lymphocytes after SARS-CoV-2 infection indicates the inhibition of body's cellular immune responses by the virus. $12 \%$ of patients also showed reduced platelet counts. All infection related biomarkers are also increased than normal levels, including serum ferritin levels in infected individuals (Chen et al., 2020).

Initially, the patients show increased concentrations of plasma IL-7, IL-8 (CXCL8), IL-9, IL-10, IL1B, IL1RA, basic FGF, TNF $\alpha$, VEGF, PDGF, IFN $\gamma$, GCSF, GMCSF, MIP1A (CCL3), MIP1B (CCL4), MCP1 (CCL2), and IP10 (CXCL10) (Huang et al., 2020). The entry of virus at a local site, like the lungs, initiates the process of inflammation which induces this cytokine storm and spreads in the whole body via systematic circulation. This cytokine storm due to infection in lungs alveoli and systematic circulation results in acute lung injury (Tisoncik et al., 2012). Patients also have a prolonged prothrombin time. In severe cases, Ddimer, blood urea, neutrophil count, and creatinine levels increase continuously, and lymphocyte count continues to decrease until death occurs. The elevating levels of neutrophils may be due to virus invasion and cytokines production (Wang et al., 2020).

\section{LIVER}

Liver enzyme levels are commonly deranged with SARSCoV-2 infection. Most commonly, patients have elevated levels of alkaline phosphatase, aspartate aminotransferase (AST) or alanine aminotransferase (ALT) and lactate dehydrogenase (LDH). The levels of these enzymes increase mostly after hospitalization. In a case report of a patient infected with COVID-19 infection, elevated levels of these enzymes were observed on day- 5 of hospitalization (Lofy et al., 2020). Patients admitted in the ICU have greater abnormal values of liver enzymes as compared to non-ICU patients. These laboratory findings are similar to SARS$\mathrm{CoV}$ and MERS-CoV, both of which cause hepatic damage (Wang et al., 2020). Due to hepatic involvement, coagulation pathway is also disturbed, leading to elevated prothrombin time (PT) and increased levels of D-dimer. Similarly, D-dimer and prothrombin time (PT) levels increase in patients admitted in ICU. These findings suggest that SARS-CoV-2 is associated with hepatic damage and cause coagulation pathway activation (Chen et al., 2020). There is little evidence to suggest that SARS-CoV-2 directly invaded the hepatic tissue. In infected patients, liver dysfunction is likely a consequence of cytokine reaction and acute inflammatory response. Presently, there is no evidence of co-infection with any hepatitis virus that can led to worse clinical outcomes, as was in the cases of SARS-CoV infected individuals (WHO, 2006). Damage to hepatic tissue is secondary to host infection with SARS-CoV-2 that primarily targets lung tissue. Clinically significant hepatitis has been reported in patients infected with SARS-CoV. The hepatitis was associated with lymphocytic infiltrates in the lobes of liver (Adams \& Hubscher, 2006). At present, SARS-CoV-2 associated hepatitis is not reported.

\section{KIDNEY}

SARS-CoV-2 do not cause direct injury to kidneys, however, there is evidence of renal damage. Kidney involvement is evident by higher levels of blood urea and creatinine in patients with COVID-19. Patients also present with acute renal injury. In a study involving 99 cases of patients with SARS-CoV-2 infection, 3\% patients presented with acute renal injury (Chen et al., 2020). With the progression of disease in severely ill-patients admitted to ICU, the clinical status of the patients rapidly deteriorates, and the levels of creatinine and blood urea progressively elevate before death (Figure 2) (Wang et al., 2020).
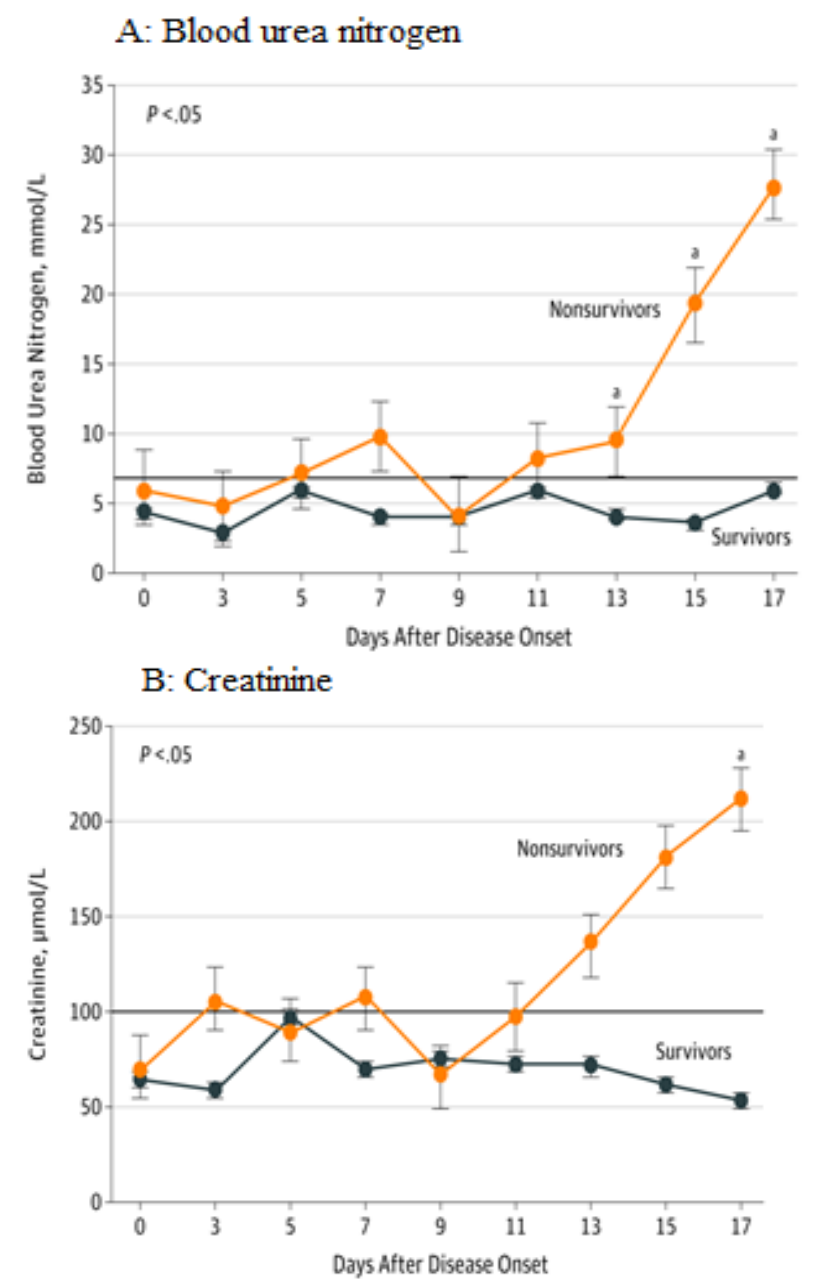

Fig. 2. Dynamic profile of blood urea nitrogen and creatinine levels in 33 patients ( 28 survivors and 5 non-survivors) infected with $2019-n \mathrm{CoV}$ infection every day after the onset of illness. The solid line in black indicates the upper normal range of each parameter, and solid line in orange shows the lower normal range of lymphocytes count. Adopted from Wang et al., 2020.

The acute renal injury in COVID-19 patients might have been linked to the viral invasion, hypoxia, and shock. Although, mortality rates are largely associated with acute respiratory failure, but lungs are not the only associated organ with the infection. Among patients with SARS-CoV-2 infection, acute renal dysfunction is also an outcome related to infection. Acute renal failure was also reported in a significant number of patients infected with SARS-CoV (Chu et al., 2005). 


\section{PREGNANCY AND COVID-19}

Pregnant females can present with fever without chills within a range of $36.5-38.8^{\circ} \mathrm{C}$, cough, myalgias, malaise, sore throat, shortness of breath, pre-eclampsia, and other symptoms of upper respiratory tract infections (Chen et al., 2020). The patient can also present with obvious gastrointestinal symptoms. The clinical picture of pregnant females infected with SARS-CoV-2 is similar to those of non-pregnant individuals infected with SARS-CoV-2. Laboratory findings include lymphopenia, elevated Creactive protein, aspartate aminotransferase (AST), and alanine aminotransferase (ALT). Chest CT scan revealed multiple patchy ground-glass shadows in lungs (Figure 3). The infection among pregnant females can lead to several complications including premature rupture of the membrane and fetal distress. There is currently no report of mortality among pregnant females infected with SARS-CoV-2 (Chen et al., 2020).
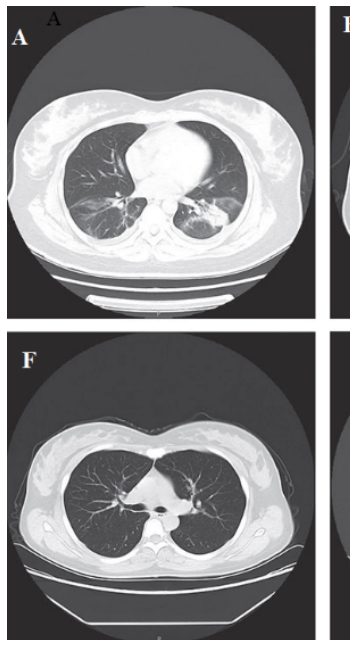
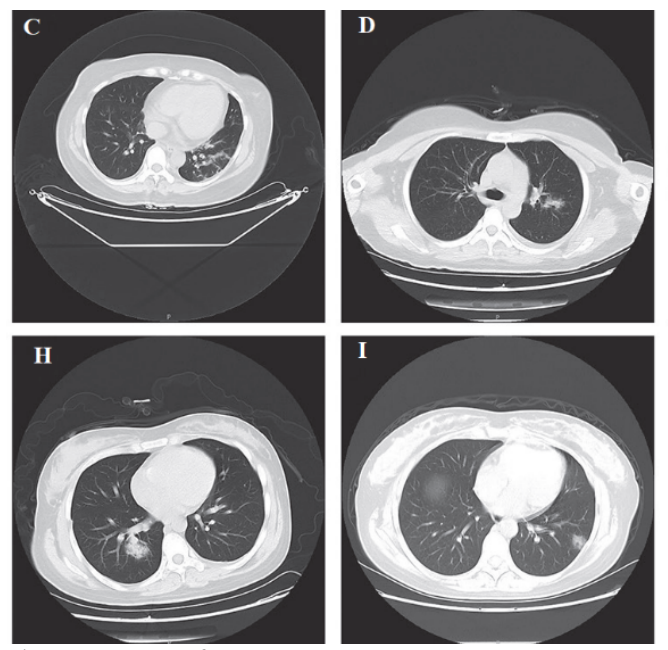
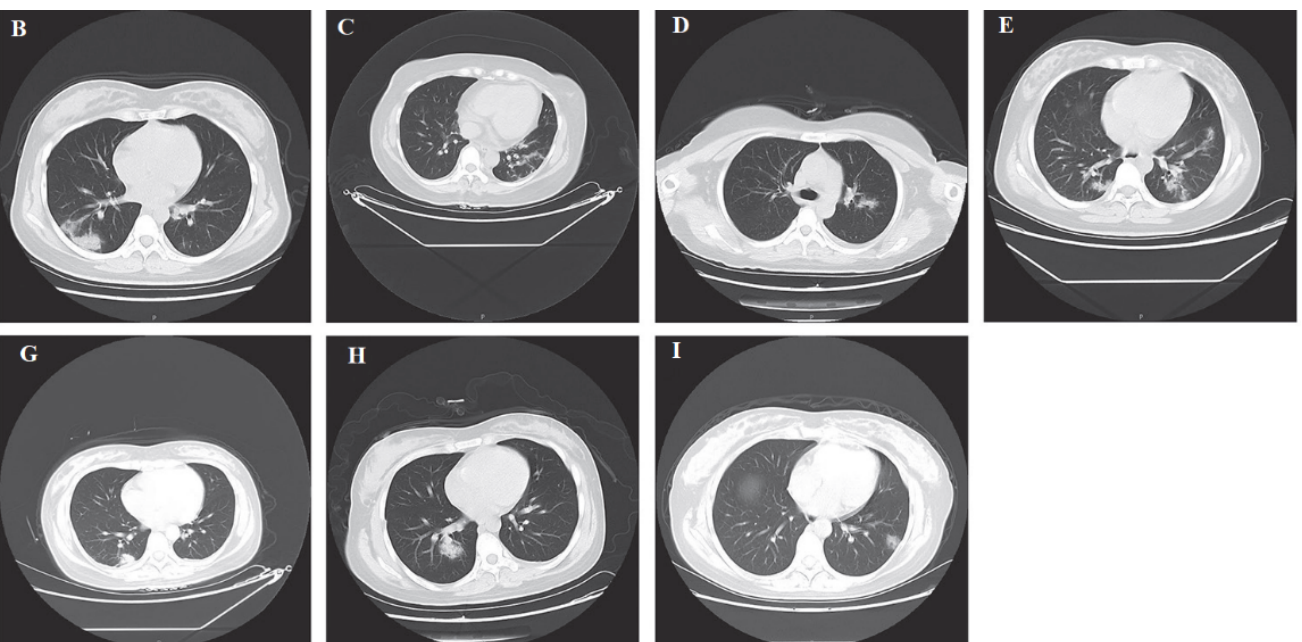

Fig. 3. Chest CT scans of 9 pregnant women

(A) Patient 1: multiple bilateral ground-glass opacities and left-sided patchy consolidation. (B) Patient 2: subpleural patchy consolidation in the right lung and slightly infiltrated shadows around left bronchus. (C) Patient 3: bilateral multiple ground-glass opacities. (D) Patient 4: patchy ground-glass opacity on left side. (E) Patient 5: bilateral multiple ground-glass opacities. (F) Patient 6: bilateral clear lung fields without obvious ground-glass opacities. (G) Patient 7: right-sided subpleural patchy consolidation. $(\mathrm{H})$ Patient 8: multiple ground-glass opacities bilaterally. (I) Patient 9: multiple ground-glass opacities bilaterally. Adopted from Chen et al., 2020.

Pregnant females are in an immunosuppressive condition and are susceptible to respiratory pathogens and developing severe pneumonia. Previous studies showed that the mortality rate was $25 \%$ among pregnant females infected with SARS-CoV and $33 \%$ of pregnant females infected with SARS-CoV required mechanical ventilation (Wong et al., 2004). However, current observations of pregnant females with SARS-CoV-2 do not follow the same pattern of SARS$\mathrm{CoV}$ infection, but we should be alert about the chances of the SARS-CoV-2 disease of following similar disease course. In a study by Chen et al. (2020) involving nine pregnant females, all women underwent caesarean section, as there was uncertainty of vertical transmission of virus via vaginal delivery. Other indications included a history of caesarean sections, fetal distress and severe pre-eclampsia. (Table 1). Special pediatric treatment was not needed in the case of any neonate.

TABLE I: NEONATAL OUTCOMES IN 9 PREGNANT WOMEN. ADOPTED FROM CHEN ET AL., 2020

\begin{tabular}{|c|c|c|c|c|c|c|c|c|c|c|}
\hline & Patient 1 & Patient 2 & Patient 3 & Patient 4 & Patient 5 & Patient 6 & Patient 7 & Patient 8 & Patient 9 & n (\%) \\
\hline $\begin{array}{c}\text { Gestational age at } \\
\text { delivery }\end{array}$ & $\begin{array}{l}37 \text { weeks, } \\
2 \text { days }\end{array}$ & $\begin{array}{l}38 \text { weeks, } \\
3 \text { days }\end{array}$ & 36 weeks & $\begin{array}{l}36 \text { weeks, } \\
2 \text { days }\end{array}$ & $\begin{array}{l}38 \text { weeks, } \\
1 \text { day }\end{array}$ & $\begin{array}{l}36 \text { weeks, } \\
3 \text { days }\end{array}$ & $\begin{array}{l}36 \text { weeks, } \\
2 \text { days }\end{array}$ & 38 weeks & $\begin{array}{l}39 \text { weeks, } \\
4 \text { days }\end{array}$ & \\
\hline Birthweight (g) & 2870 & 3730 & 3820 & 1880 & 2970 & 3040 & 2460 & 2800 & 3530 & \\
\hline $\begin{array}{c}\text { Low birthweight } \\
(<2500 \mathrm{~g})\end{array}$ & No & No & No & Yes & No & No & Yes & No & No & $2(22 \%)$ \\
\hline Premature delivery & No & No & Yes & Yes & No & Yes & Yes & No & No & $4(44 \%)$ \\
\hline $\begin{array}{c}\text { Apgar score (1 min, } 5 \\
\text { min) }\end{array}$ & 8,9 & 9,10 & 9,10 & 8,9 & 9,10 & 9,10 & 9,10 & 9,10 & 8,10 & \\
\hline $\begin{array}{c}\text { Severe neonatal } \\
\text { asphyxia }\end{array}$ & No & No & No & No & No & No & No & No & No & 0 \\
\hline Neonatal death & No & No & No & No & No & No & No & No & No & 0 \\
\hline Fetal death or stillbirth & No & No & No & No & No & No & No & $\mathrm{No}$ & No & 0 \\
\hline
\end{tabular}

After birth the neonates were tested to determine the possibility of intrauterine SARS-CoV-2 fetal infection. Neonatal throat swab samples, amniotic fluid and cord blood samples all tested negative for SARS-CoV-2, proving no intrauterine fetal infections occurred (Chen et al., 2020).
These findings are similar to SARS-CoV infection. Previous studies indicated no evidence of transmission of SARS-CoV form mother to fetus during birth (WHO, 2006). However, on Feb 6, 2020, a neonate born to a pregnant female infected with 2019-nCoV pneumonia tested positive for SARS-CoV- 
2 infection 36 hours after birth, but the major clinical details of this case are not available. SARS-CoV-2 has a potential for vertical transmission in pregnant women and there is a need for evaluating it further (Chen et al., 2020).

\section{CONCLUSION}

Clinical manifestations of COVID-19 are diverse, mimicking those of pneumonia and commonly presenting with fever, cough, myalgias, fatigue, and less commonly with diarrhea and vomiting. Presenting symptoms in pregnant females are also similar to those of non-pregnant females. COVID-19 infection is confirmed by radiologic and laboratory findings. The pneumonia progressively develops into severe acute respiratory infection, acute respiratory distress syndrome, acute respiratory failure, hepatic injury, kidney failure and cardiovascular complications. Prompt administration of antibiotics in persons with reduced immune function, such as diabetics, older people, patients with HIV, patients using immunosuppressive drugs, and pregnant females strengthen immune function reducing complications and mortality. Patients with underlying chronic diseases show poor outcomes with treatment and result in severe and fatal diseases.

\section{REFERENCES}

Adams, D. H., \& Hubscher, S. G. (2006). Systemic Viral Infections and Collateral Damage in the Liver. 1057-1059. https://doi.org/10.2353/ajpath.2006.051296

Centers for Disease Control and Prevention (CDC). 2020. Coronavirus Disease 2019 (COVID-19) Situation Summary. Retrieved from https://www.cdc.gov/coronavirus/2019-ncov/summary.html

Chen, H., Guo, J., Wang, C., Luo, F., Yu, X., Zhang, W., ... Zhang, Y. (2020). Clinical characteristics and intrauterine vertical transmission potential of COVID-19 infection in nine pregnant women: a retrospective review of medical records. The Lancet, 6736(20), 1-7. https://doi.org/10.1016/S0140-6736(20)30360-3

Chen, N., Zhou, M., Dong, X., Qu, J., Gong, F., Han, Y., .. Yu, T. (2020). Epidemiological and clinical characteristics of 99 cases of 2019 novel coronavirus pneumonia in Wuhan, China: a descriptive study. The Lancet, 395(10223), 507-513. https://doi.org/10.1016/S01406736(20)30211-7

Chu, K. H., Tsang, W. K., Tang, C. S., Lam, M. F., Lai, F. M., To, K. F., .. Lai, K. N. (2005). Acute renal impairment in coronavirus-associated severe acute respiratory syndrome. Kidney International, 67(2), 698705. https://doi.org/10.1111/j.1523-1755.2005.67130.x

Gorbalenya, A.E., Baker, S.C., Baric, R.S. et al. The species Severe acute respiratory syndrome-related coronavirus: classifying 2019-nCoV and naming it SARS-CoV-2. Nat Microbiol (2020). https://doi.org/10.1038/s41564-020-0695-Z

Guan, W., Ni, Z., Hu, Y., Liang, W., Ou, C., He, J., ... Zhong, N. (2020). Clinical characteristics of 2019 novel coronavirus infection in China. MedRxiv. https://doi.org/10.1101/2020.02.06.20020974

Holshue, M. L., DeBolt, C., Lindquist, S., Lofy, K. H., Wiesman, J., Bruce, H., ... Pillai, S. K. (2020). First Case of 2019 Novel Coronavirus in the United States. New England Journal of Medicine. https://doi.org/10.1056/nejmoa2001191

Huang, C., Wang, Y., Li, X., Ren, L., Zhao, J., Hu, Y., ... Gu, X. (2020) Clinical features of patients infected with 2019 novel coronavirus in Wuhan, China. 497-506. https://doi.org/10.1016/S01406736(20)30183-5

Lofy, K. H., Wiesman, J., Bruce, H., Spitters, C., Ericson, K., Wilkerson, S., ... Pillai, S. K. (2020). First Case of 2019 Novel Coronavirus in the United States. The New England Journal of Medicine, 1-9. https://doi.org/10.1056/NEJMoa2001191

Lu, R., Zhao, X., Li, J., Niu, P., Yang, B., Wu, H., ... Tan, W. (2020). Genomic characterization and epidemiology of 2019 novel coronavirus: implications for virus origins and receptor binding. Lancet (London, England), 6736(20), 1-10.
https://doi.org/10.1016/S0140-6736(20)30251-8

Tisoncik, J. R., Korth, M. J., Simmons, C. P., Farrar, J., Martin, T. R., \& Katze, M. G. (2012). Into the Eye of the Cytokine Storm. 16-32. https://doi.org/10.1128/MMBR.05015-11

Wan, Y., Shang, J., Graham, R., Baric, R. S., \& Li, F. (2020). Receptor recognition by novel coronavirus from Wuhan: An analysis based on decade-long structural studies of SARS. Journal of Virology. https://doi.org/10.1128/jvi.00127-20

Wang, D., Hu, B., Hu, C., Zhu, F., Liu, X., Zhang, J., ... Peng, Z. (2020). Clinical Characteristics of 138 Hospitalized Patients with 2019 Novel Coronavirus-Infected Pneumonia in Wuhan, China. JAMA - Journal of the American Medical Association. https://doi.org/10.1001/jama.2020.1585

World Health Organization. (2020). Briefing on COVID-19. Retrieved from https:/www.who.int/dg/speeches/detail/who-director-general-sopening-remarks-at-the-media-briefing-on-covid-19---9-march-2020

World Health Organization. WPRO. (2006). SARS-how a global disease stopped. ISBN 9290612134

Wong, S. F., Chow, K. M., Leung, T. N., Ng, W. F., Ng, T. K., Shek, C. C., ... Tan, P. Y. H. (2004). Pregnancy and perinatal outcomes of women with severe acute respiratory syndrome. American Journal of Obstetrics and https://doi.org/10.1016/j.ajog.2003.11.019.
Gynecology. 